\title{
Electrical conductivity sensors for water penetration monitoring in building masonry materials
}

\author{
M. Guizzardi · D. Derome · D. Mannes • \\ R. Vonbank $\cdot$ J. Carmeliet
}

Received: 8 January 2015 / Accepted: 29 June 2015/Published online: 30 September 2015

(C) RILEM 2015

\begin{abstract}
Water penetration in porous building materials can cause damage in building components exposed to wet environmental conditions. A system to monitor water penetration by means of electrical conductivity measurements is presented (Gummerson et al. Build Environ 15:101-108, 1980). The sensor allows non-destructive and continuous monitoring of different layers of building envelopes with high space and time resolution. Capillary uptake experiments have been performed to assess the reliability of the developed system. Sensors data are compared to the results of the analysis of neutron radiographs taken during the experiments. These sensors are easy to apply in different positions, even deep in the structural components where direct gravimetric investigation is not possible. Electrical conductivity measurements provide a good temporal description of the capillary uptake process, however a calibration for moisture content quantification could not yet be achieved.
\end{abstract}

M. Guizzardi $(\bowtie) \cdot$ J. Carmeliet

ETH Zürich, Eidgenössische Technische Hochschule,

Zurich, Switzerland

e-mail: michelaguizzardi@gmail.com

M. Guizzardi · D. Derome - R. Vonbank

Empa, Swiss Federal Institute for Materials, Science \&

Technology, Dübendorf, Switzerland

D. Mannes

PSI, Paule Scherrer Institute, Villigen, Switzerland
Keywords Moisture content sensor - Capillary uptake $\cdot$ Electrical conductivity $\cdot$ Neutron radiography

\section{Introduction and problem statement}

Water penetration in porous building materials exposed to environmental conditions can lead to damage like freeze/thaw cycles, concrete spallation, mold and rot fungi growth, salt transport and consequently crystallization. An in situ reliable mean to monitor water penetration in real structures is missing. Such mean would be helpful for the long-term performance assessment of buildings, the assessment of retrofit and renovation strategies of building envelopes, the evaluation of the need of dehumidification treatments, etc. In this paper, a moisture detection system based on electrical conductivity is presented. The system allows repeatable measurements at different positions and at different times without affecting the transport process, a clear advantage over gravimetric measurements of material cutouts. The system is here tested on clay bricks and wetapplied mortars and plasters through an experimental campaign and results are compared with neutron radiography measurements. Firstly a short overview of available measurement techniques is given, then the experimental set-up and procedures are described. The system performance is assessed and the possibility of practical applications of this monitoring system is evaluated. 
The standard procedure to determine moisture content in a porous material is the gravimetric method. It consists in weighing a material sample at a given moist state and at dry state, the gravimetric moisture content being the mass difference between these two states with respect to the dry mass. Moisture content profiles within a material can also be determined by slicing samples and taking the moisture content of the slices separately. The method is very simple but poses some disadvantages such as it is laborious, time consuming, destructive and thus not repeatable. Furthermore it does not allow the monitoring of transient conditions and its space resolution is limited. Some methods to monitor moisture content changes in masonry through repeated gravimetric measurements have been proposed, but are difficult to apply in walls with exterior finishing [21].

To address these limitations, non-destructive and time-resolved procedures to monitor moisture transport and accumulation in porous materials have been developed; reviews of methods are given in [12] and in [14]. Such methods can be divided in (1) hygrothermal probes, (2) radiation techniques, and (3) methods measuring the variation of a property influenced by moisture content. For example, probes of the first type measure the relative humidity in holes drilled in the material and, then, assuming the air being in hygrothermal equilibrium with the surrounding wet material, the moisture content can be derived from sorption isotherms.

The second group of measurement methods is based on radiation attenuation. For example, the attenuation of $\mathrm{X}$-rays depends on the density and atomic number of the irradiated material. However, as water has a much lower X-ray attenuation coefficient compared to other building materials, it is challenging to get high resolution moisture content results with this technique. Other methods are based on $\gamma$-rays or neutron irradiation. Neutrons have a much higher interaction probability with hydrogen compared to $\mathrm{X}$-rays and neutron imaging provides an accurate way to spatially quantify the presence of water in materials. Nuclear magnetic resonance measures moisture content in porous materials with high accuracy at high measuring speed and low sensitivity to temperature. It selectively probes the hydrogen nuclei distinguishing between free, physically bound and chemically bound water. However complications can arise due to the presence of large amount of paramagnetic ions, as in the case of building materials like bricks and mortars [16]. Methods easier to apply in situ rely on ultrasound, as sound absorption and its propagation speed depend on water content [2], microwave [13] or ground penetrating radar (GPR) [5]. However, these three methods are not precise, especially ultrasound measurement and do not provide sufficient spatial resolution [12]. The third class of measurement methods uses the dependence of a material property on moisture content, such as dielectric permittivity and thermal or electrical conductivity. For example, capacitance sensors, also known as dielectric sensors, are used to estimate the water content of soils in petroleum engineering and soil science applications [6]. An example of their application to civil or building engineering is given in [4].

Electrical conductivity is a bulk material property defined as the current flowing through a sectional area divided by the voltage measured across a unit cube length and is expressed in Siemens per meter $(\mathrm{S} / \mathrm{m})$. Resistivity is the inverse of conductivity and is expressed in $\mathrm{Ohm}$ meter $(\Omega \mathrm{m})$. It is frequently assumed that the electrical conductivity has contributions from both the solid and the liquid phases. The solid term is considered independent of moisture content except under extremely dry conditions. The second term, attributed to the fluid in the pore space, is the product of the conductivity of the fluid, its content and a transmission coefficient that is itself a linear function of water content [7]. Electrical conduction is largely electrolytic and can vary by several orders of magnitude depending on porosity, hydraulic permeability (pore connection), moisture content, concentration of dissolved electrolytes, temperature and phase of the pore fluid and the amount and composition of colloids, i.e. clay content. More specifically, as an example of affecting parameters, the electrical conductivity of mortar will depend on cement type and content, water-to-cement ratio, curing conditions, degree of hydration, temperature, aggregate type and gradation. With all these factors at play, it is challenging to have a unique relationship between moisture content and resistivity, as demonstrated in work on concrete (e.g. [15, 20]).

As mentioned, electric conductivity is largely electrolytic, i.e. the electrical current is transported by the ions moving in the water solution. Distilled water does not contain dissolved salts and, as a result, has a very low electrical conductivity (equal to 
$\left.5.5 \times 10^{-6} \mathrm{~S} / \mathrm{m}\right)$. The electrical conductivity increases as the concentration of ions increases. The electrical conductivity of the water also increases with increasing water temperature.

As an example for porous materials, an empirical relationship is used for most rocks to describe the relation between porosity and electrical conductivity, a relationship referred to as Archie's formula [1]:

$$
C_{m}=\frac{1}{a} \times C_{w}^{m} \times S_{w}^{n}
$$

where $C_{m}$ is the electrical conductivity of the rock with fluid, $C_{w}$ the electrical conductivity of the fluid, $S_{w}$ the degree of saturation, $m$ the cementation exponent of the rock (usually in the range 1.8-2.0 for sandstones), $n$ the saturation exponent (usually close to 2 ) and $a$ the tortuosity factor. This empirical law was developed for ionic flow in clean, consolidated sands where electrical conduction is assumed to take place only in the fluid phase. This law was initially used to estimate oil reserves, nevertheless has found a wide application for the characterization of porous materials, including soil and construction materials [3]. Moisture content evaluations through electrical conductivity measurements are used, e.g. also in petroleum engineering and soil science [17], while in building physics applications of this technique for moisture content measurements in laboratory tests with concrete are described in $[18,19]$ : electrical conductivity is analyzed and the contribution of factors, such as temperature, relative humidity and conductivity of the pore solution, is seen.

Therefore, the goal of our work consists in testing a simple electrical conductivity measurement device that allows no-destructive and continuous measurements in different commonly used building materials with a refined spatial resolution, in order to provide, not a moisture content quantification as attempted in previous works, but a qualitative description of water front movements in deepest and non-accessible layers of building structures.

\section{Sensor design and fabrication}

Moisture content sensors using electrical conductivity measurement are designed and integrated in building materials, bricks, mortar and plasters, with a different solution for voltage application and resistance measurement.

\subsection{Instrumentation of clay bricks}

For the monitoring of water penetration in bricks, voltage is applied across the brick through couples of tin contacts drilled into the brick. Two lines of holes with a diameter of $8 \mathrm{~mm}$ (five or six holes in different brick sensor prototypes) are drilled at the two opposite faces of the brick at a distance of $34 \mathrm{~mm}$ from each other (Fig. 1a). After drilling, the holes are carefully cleaned of dust with compressed air and then filled with melted tin (Fig. 1b). After filling, the tin is re-melted with a "melting pen" in order to create a better contact between the metal and the surface of the hole and remove eventual air bubbles formed during tin pouring. Finally, wires connecting the contacts to the electrical circuit are inserted in the tin (Fig. 1c). For demonstration, Fig. 1d shows a sawn section of the brick sensor with sectioned tin contacts. Wires apply the electrical current through the material and are used to measure the electrical resistance of the materials in the position between the two contacts. Different positions and more measurement points could be drilled according to different needs and experimental goals. The brick, which arrived dried from the kiln, is kept at constant conditions of $21^{\circ} \mathrm{C}$ and $50 \% \mathrm{RH}$ until the experiment.

\subsection{Sensor for mortar and plaster applications}

To perform electrical conductivity measurements in wet-applied building materials, such as mortars and plasters, a device is designed to be placed in position so that the wet material enters and fills the voids between the couple of contacts before curing (Fig. 2). The plastic device is equipped with 10 measurement positions consisting on couples of copper contacts $3 \mathrm{~mm}$ apart from each other. Once the material is cured (thus after more than 56 days of exposure in $80 \% \mathrm{RH}$ environment), the electrical resistance across the material at the 10 positions can be measured. The height of the sensor is here of $3 \mathrm{~cm}$, but the shape of the sensors can be optimized to suit specific applications or dimensions of other mixtures, aggregates or layers.

\section{Experimental set up for sensors verification}

The experiment consists in subjecting 4 different materials to water uptake while monitoring the moisture content with neutron radiography and measuring the 
(a)

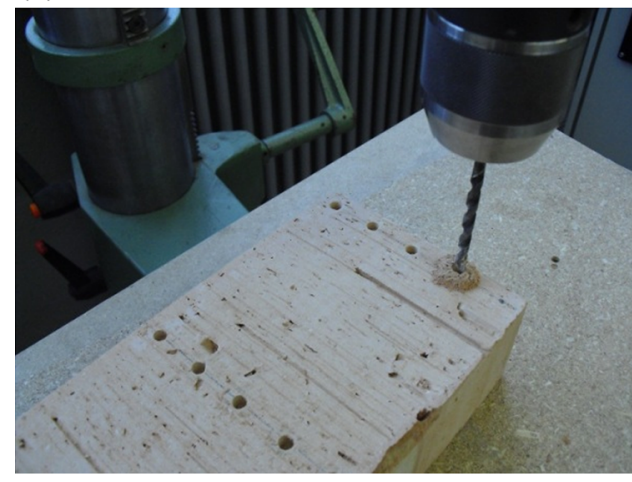

(c)

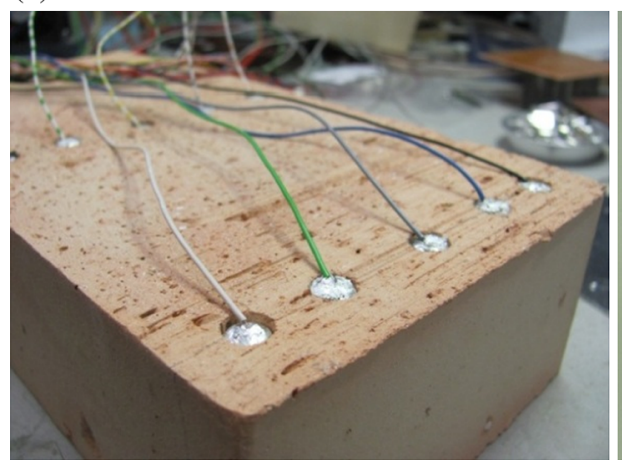

(b)

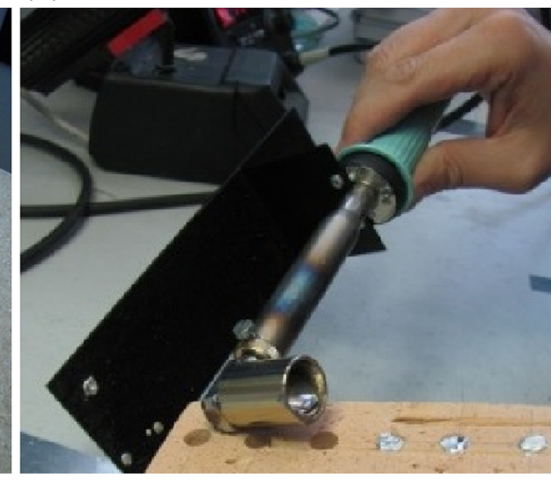

(d)

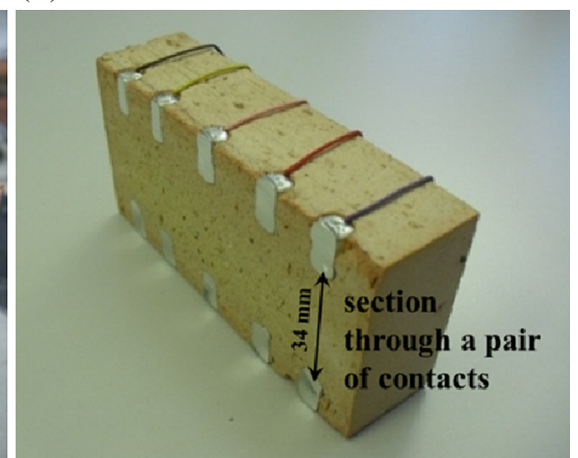

Fig. 1 Photos of the instrumentation steps of the brick: precise drilling of holes (a), filling of holes with melted tin (b) and connection of electrical wires (c). Section of a brick sensor to demonstrate the position of the tin contacts (d)

electrical conductivity at different heights in the samples using the above mentioned sensors.

\subsection{Materials}

The performance of the moisture content sensors is verified for the following four materials: clay brick, lime-cement render, cement mortar and aerogel plaster. The clay brick is a common full brick. The limecement render, cement mortar and the aerogel are products available on the market, specifically F662, F920 and F220 from the manufacturer Fixit. The cement mortar is a common cement based mortar commonly used in masonry construction. The binder of the lime-cement render is a mixture of hydraulic lime, white slaked lime and cement, with crushed and round limestone sand grains of less than $4 \mathrm{~mm}$. The aerogel plaster is an insulation render developed in our laboratory in collaboration with the industrial partner. This lightweight mortar $\left(200 \mathrm{~kg} / \mathrm{m}^{3}\right)$ can be applied both as external and internal render. It consists of hydrophobized granular silica aerogel (60-90 vol\%) and lime-based binder. The use of the aerogel aggregates confers high thermal resistance and low density to the material, making it suitable for thermal insulation applications. The thermal conductivity of the aerogel plaster is $25 \pm 2 \mathrm{~mW} / \mathrm{mK}$, which is lower than the thermal conductivity of common renderings which is around $65 \mathrm{~mW} / \mathrm{mK}$. The main hygrothermal properties of the materials are determined in the laboratory and summarized in Table 1, where vapor resistance factor is the ratio of the material to air water vapor diffusion coefficient, capillary absorption coefficient is the slope of liquid water uptake versus square root of time, sorption isotherm refers to vapor uptake at different relative humidity and capillary saturation moisture content is the maximum amount of uptaken liquid under atmospheric conditions.

\subsection{Samples and experimental setup}

Brick samples of $16 \mathrm{~mm}$ thickness are equipped with six pairs of holes that have been drilled along the $12 \mathrm{~cm}$ height of the sample at 1, 3, 5, 7, 9 and $11 \mathrm{~cm}$ 
from the brick/water interface, as shown in Fig. 3. The mortar samples are equipped with two electrical conductivity sensors in a row, allowing the measurement of electrical conductivity at 20 positions, at $3 \mathrm{~mm}$ interval, for a total length of $6 \mathrm{~cm}$ along the direction of water front penetration (Fig. 4a). The wetapplied material samples are made using a mold where the mixture is poured to a $2 \mathrm{~cm}$ layer. An aluminum foil, which is transparent to neutron, lines the mold and remains on the samples, providing side sealing during

(a)

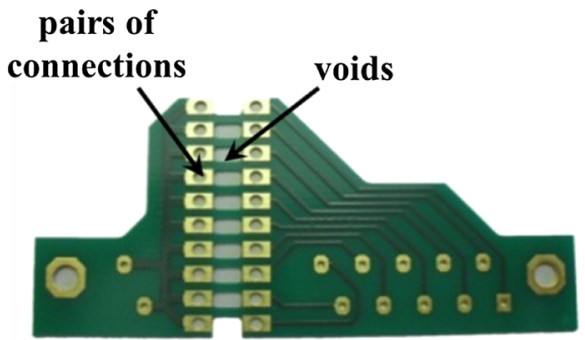

(b)

(c)

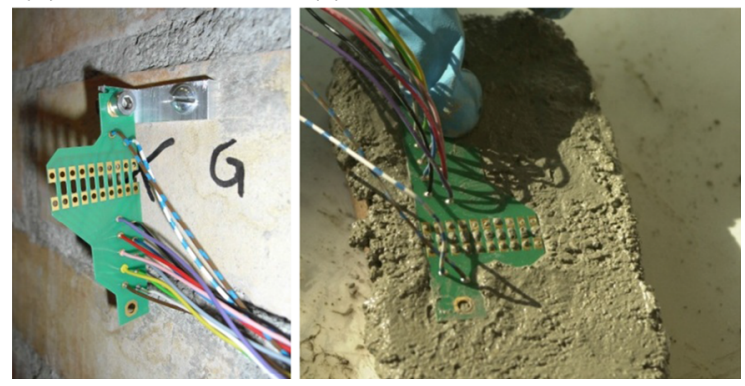

Fig. 2 Photos of moisture content sensor developed for wetapplied materials (a); positioning on the exterior surface of a masonry wall, before the application of a render layer (b) moisture content sensor set into a fresh mortar masonry joint (c) the uptake experiments. Thermocouples are inserted at every second contact in order to control possible effects of temperature on the sensors readings. Once poured in the frames, the samples are initially cured at $80 \%$ relative humidity to limit initial shrinkage as this would compromise the adherence between the electrical contacts and the material.

The samples are then subjected to capillary uptake. Such test is standard and consists in recording the mass of moisture uptake in the sample at defined time intervals after the sample is put in contact with a pool of water. According to Washburn law, the total mass of uptaken water increases linearly with square root of time and the capillary absorption coefficient is defined as the slope of the curve.

The instrumented samples are installed in a frame where a tank is filled with water until contact with the bottom of the sample (see Fig. 5). All instrumentation is connected to a Agilent 34970A Data Acquisition Switch Unit. The total mass of the samples is measured before and after the experiment. During the capillary uptake experiment, measurement is performed at each electrical pair. A DC current of $50 \mu \mathrm{A}$ is applied across a circuit of 2 resistances in parallel, one being an electrical pair and the other a resistance of $100 \mathrm{M} \Omega$. A voltage between 0 and $5 \mathrm{~V}$ is measured, allowing the determination of the electrical conductance. As explained below, the direction of the current is reversed at every measurement. Data from the electrical conductivity sensors is continuously recorded. All along the actual experiment, neutron radiographies are recorded at every $2 \mathrm{~min}$, while the

Table 1 Measured material properties of clay brick, cement mortar, cement mortar and aerogel plaster

\begin{tabular}{|c|c|c|c|c|c|}
\hline & \multicolumn{2}{|c|}{ Clay brick } & Cement mortar & Lime cement mortar & Aerogel plaster \\
\hline Density & \multicolumn{2}{|c|}{$1553 \mathrm{~kg} / \mathrm{m}^{3}$} & $1623 \mathrm{~kg} / \mathrm{m}^{3}$ & $1668 \mathrm{~kg} / \mathrm{m}^{3}$ & $200 \mathrm{~kg} / \mathrm{m}^{3}$ \\
\hline Thermal conductivity & \multicolumn{2}{|c|}{$0.684 \mathrm{~W} / \mathrm{mK}$} & $0.633 \mathrm{~W} / \mathrm{mK}$ & $0.464 \mathrm{~W} / \mathrm{mK}$ & $0.027 \mathrm{~W} / \mathrm{mK}$ \\
\hline Vapor resistance factor & \multicolumn{2}{|c|}{$14-15$} & $17-21$ & 11 & 4 \\
\hline Capillary absorption coefficient & \multicolumn{2}{|c|}{$0.115 \mathrm{~kg} / \mathrm{m}^{2} \mathrm{~s}^{0.5}$} & $0.023 \mathrm{~kg} / \mathrm{m}^{2} \mathrm{~s}^{0.5}$ & $0.077 \mathrm{~kg} / \mathrm{m}^{2} \mathrm{~s}^{0.5}$ & $0.032 \mathrm{~kg} / \mathrm{m}^{2} \mathrm{~s}^{0.5}$ \\
\hline \multirow[t]{6}{*}{ Sorption isotherm } & RH & $\mathrm{w}\left(\mathrm{kg} / \mathrm{m}^{3}\right)$ & $\mathrm{w}\left(\mathrm{kg} / \mathrm{m}^{3}\right)$ & $\mathrm{w}\left(\mathrm{kg} / \mathrm{m}^{3}\right)$ & $\mathrm{w}\left(\mathrm{kg} / \mathrm{m}^{3}\right)$ \\
\hline & 0 & 0 & 0 & 0 & 0 \\
\hline & 0.3 & 1.4 & 2.44 & 4.2 & 1.1 \\
\hline & 0.5 & 1.9 & 5.56 & 17.9 & 2.55 \\
\hline & 0.8 & 10.5 & 20 & 50.5 & 10 \\
\hline & 0.95 & 17.1 & 113.2 & 59.7 & 43.4 \\
\hline Capillary saturation moisture content & 303.8 & & 148 & 286.8 & 400 \\
\hline
\end{tabular}


Fig. 3 Drawing (a) and photo (b) of the instrumented brick used in the experimental campaign with neutron radiography, with dimensions of the sample and position of the electrical connections

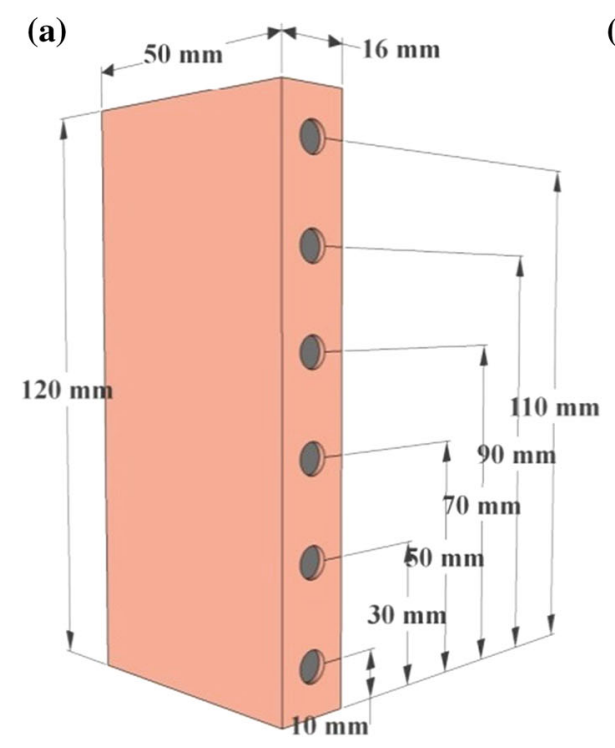

(b)

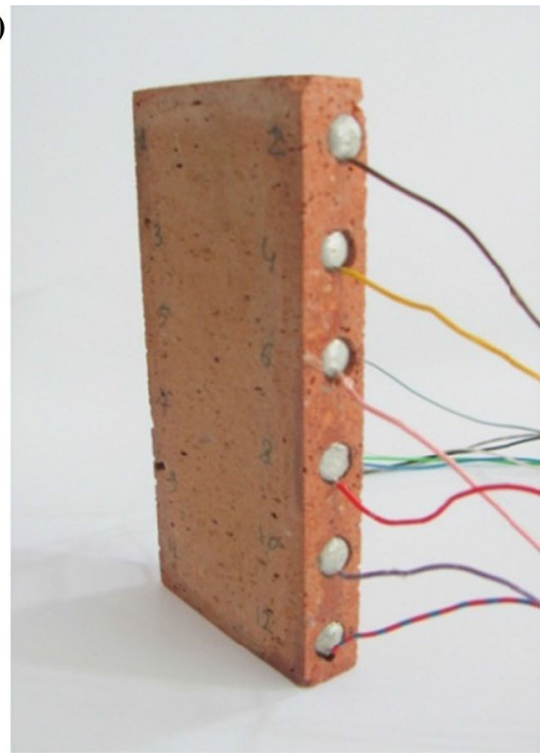

(a)

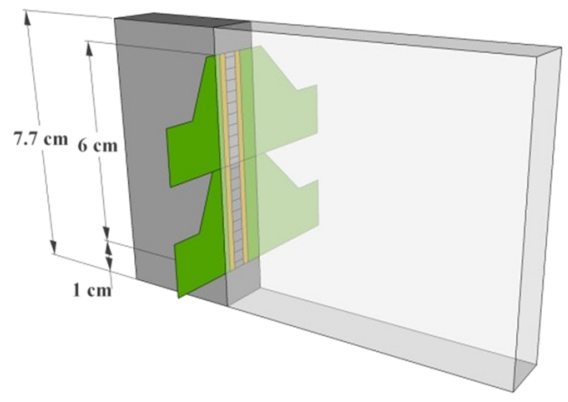

(b)

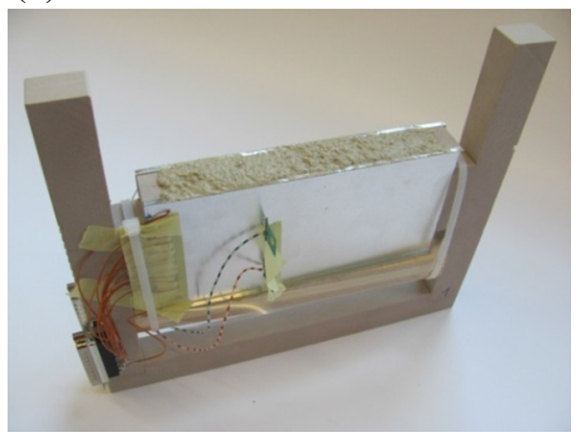

Fig. 4 a, b Drawing (a) and photo (b) of the wet-applied material sample used in the experiment with the neutron radiography

(a)

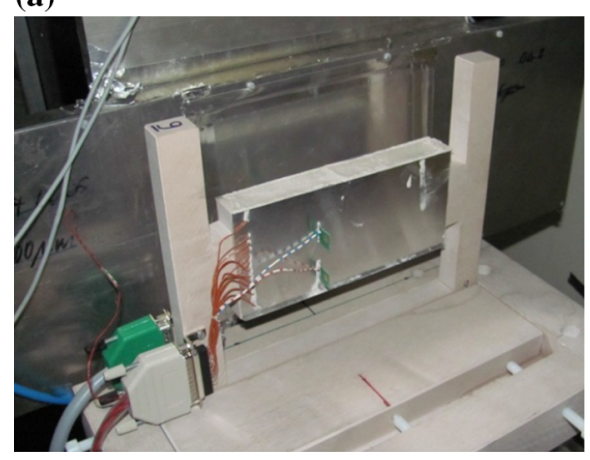

(b)

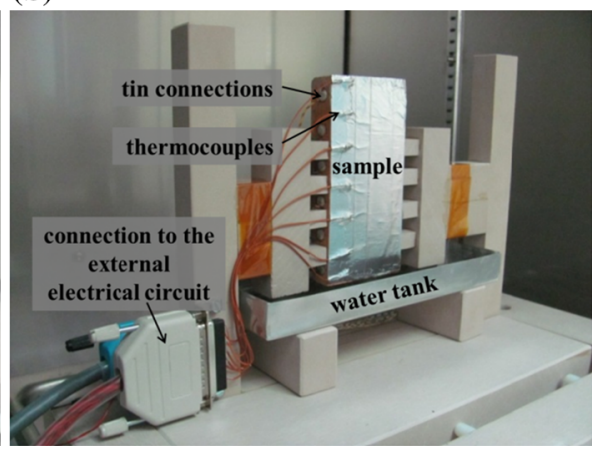

Fig. 5 Photos of the experimental set-up used in the experimental campaign with neutron beam, with sample and position of the electrical connections, for wet-applied material (a) and brick (b) 
first radiography is acquired within 5 min of the filling of the water tank, thus the start of the experiment.

\subsection{Neutron radiography}

A neutron radiography system basically consists of a neutron beam and a detector system (Fig. 6). The investigated sample is placed in the beam and the detector captures the transmitted neutrons. At the NEUTRA (Neutron Transmission Radiography) beamline at PSI (Paul Scherrer Institute) in Villigen (Switzerland), neutrons are extracted by spallation by the Swiss Neutron Spallation Source (SINQ), then they are slowed down in a $\mathrm{D}_{2} \mathrm{O}$ filled moderator to an average energy around $25 \mathrm{meV}$ or velocities around $2200 \mathrm{~m} / \mathrm{s}$. After moderation, when neutrons are in thermal equilibrium with a moderator material, a collimator selects the neutrons with a trajectory towards the sample. Neutrons passing through the sample interact with the atomic nuclei of the investigated material, where neutrons can either be absorbed or scattered. The intensity of the transmitted beam, $I$, can be described by Beer-Lambert law:

$I=I_{0} \times e^{-\Sigma z}$

where $I_{0}$ is the intensity of the incident neutron beam, $z$ is the thickness of the sample, $\Sigma$ is the neutron linear attenuation coefficient, a macroscopic parameter of neutron attenuation by a given material.

The detector system (a scintillator screen in combination with a CCD camera) records the neutron flux going through the sample. The detector has a homogeneous thin neutron absorbing layer and has an energydependent macroscopic absorption cross section. Thus, the detector converts the neutrons into visible light photons. The scintillator, here, is a plane slab of $\mathrm{ZnS}$ crystal, doped with $6 \mathrm{Li}$ as neutron absorbing agent (100 $\mu \mathrm{m}$ thick). The photons are then led via a mirror onto a cooled 16-bit CCD camera $(1024 \times 1024$ pixels at NEUTRA). For this experiment, a spatial resolution of $85 \mu \mathrm{m} /$ pixel could be achieved for a field of view of $87 \mathrm{~mm}$ wide. The exposure time per radiograph was $8 \mathrm{~s}$ and the sample was placed $20 \mathrm{~cm}$ in front of the scintillator. The resulting digital image consists of a discrete array of numbers (grey values) for each position $i\left(x_{i}, y_{i}\right)$ at each recording time. This value is proportional to the physically registered neutron intensity. Thus, the intensity $I\left(x_{i}, y_{i}\right)$ of a pixel of a finite area
$\Delta x \times \Delta y$ of the image is proportional to the total number of detected neutrons in the pixel area. This allows the quantitative evaluation of moisture content from neutron radiographies. However, scattered neutrons can hit the detector and provide too high values. Also, the polyenergetic spectrum of the neutron beam may affect the effective cross section of the detector material used for the evaluation of the quantitative mass thickness from the measured neutron flux. For an accurate quantification, scattering and spectral effects must be corrected. To do, the characteristics of the facility used, such as the energy spectrum of the neutron beam, its intensity and its spatial distribution, together with the properties of the detector system, are taken into account. For the quantitative analysis of neutron radiography, the algorithm QNI developed at PSI [10, $11]$ is used. The water mass thickness is estimated as a single variable from QNI and the change in the moisture content $w(t)$ from the initial (dry) state is calculated. The resolution in moisture content is defined as the moisture content corresponding to one gray scale difference between wet and dry states. The resolution depends on the density and the attenuation coefficient of water, the thickness of the sample and the neutron radiograph intensity, $I_{0}$. A complete description of the principles and equations used for correction and water content determination can be found in [10, 11 and 22].

For each capillary uptake experiment, initial reference radiographs are recorded as needed for the later correction for artifacts and quantification analysis. They consist in a set of five dark current images with the beam closed, a set of five images with the neutron beam open without the sample and a set of five black body images with the sample shielded from the beam with boronated, thus neutron opaque, polyethylene blocks (Fig. 7).

\subsection{Experimental protocol}

Once the tank is filled with water and contact with the bottom of the sample is ensured, the neutron hutch is closed and neutron radiographies are acquired. Later, during the uptake, as water is depleted, the contact between the water and the sample is loss and redistribution and drying occur. Each capillary uptake experiment lasts $4-8 \mathrm{~h}$ documenting both the initial uptake phase and the following redistribution of water after water contact loss, in order to assess the results of the electrical conductivity sensors in both phases. 
Ambient temperature was not controlled but remained in the range of $24-28{ }^{\circ} \mathrm{C}$.

\section{Experimental results}

\subsection{Liquid uptake in instrumented brick}

Neutron radiography images once corrected and analyzed, as explained in Sect. 3.3, are used to plot moisture content profiles at each time step during the uptake experiments.

For the brick sample, the uptake process is documented over 264 min during which the water front travels to almost $9 \mathrm{~cm}$ above the water contact. In Fig. 8, moisture content profiles calculated from neutron radiographs during the uptake experiment are plotted with a time interval of $4 \mathrm{~min}$. The distance between profiles is decreasing with time, which indicates that the rate of the uptake decreases during the uptake. The water front does not reach the height of connection five during the experiment. It can be also observed that the final profiles, in blue lines, show decreasing moisture content values in the bottom part of the sample. As mentioned above, this is due to the loss of water/sample contact and the starting of drying in the lower portion of the brick sample and to the redistribution of water in the wet portion of the sample. In the graph, the positions of the measurement contacts are shown in dashed lines and the profiles corresponding to the arrival of the water front to each position, as detected by the electrical conductivity measurements, are highlighted in red.
From the electrical conductance profiles shown in Fig. 9a, the arrival time of the water front at each sensor position is determined as the time corresponding to the highest electrical conductivity increase. In Table 2 and Fig. 10, the times corresponding to the water front arrival at the height of each sensor connection obtained by neutron radiography are compared to the arrival times detected by the brick sensor. The figure shows good agreement between results obtained by the two methods, showing a very good performance of the electrical conductivity sensor for identifying the water front arrival times during the uptake process.

We note that the electrical conductivity curves in Fig. 9a show a steep step corresponding to the arrival time of water front at each contact positions 1,2, 3 and 4. However, after the water front passage, a decrease in the recorded values of electrical conductivity at each position can be observed. This can be to different effects other then water redistribution. In the experiment described in this paper, the electrical conductivity is measured always in the same direction and this method can induce ions migration and accumulation on one side of the sample, thus depleting the number of carriers available in the porous medium, resulting in a lower electrical conductivity. On the other hand, the liquid transport can advect ions during the capillary rise, a known phenomenon called polarization. Thus, a higher salt concentration is expected at the water front compared to downstream of the front. To eliminate the effect of the ions accumulation on one side of the sample, the measurement procedure of electrical conductivity was modified in further experiments

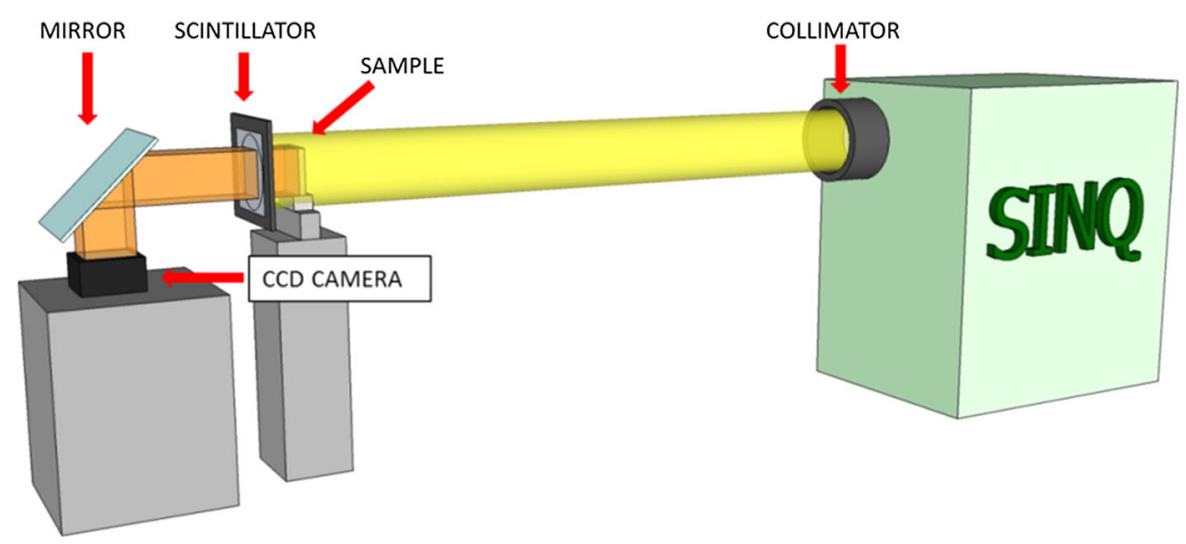

Fig. 6 Schematic representation of the NEUTRA facility 
Fig. 7 Sequence of neutron radiographs at different time steps during the capillary uptake experiment with the neutron brick. Only the central portion of the sample is visible because the lateral metal connections of the sensors are protected from the beam

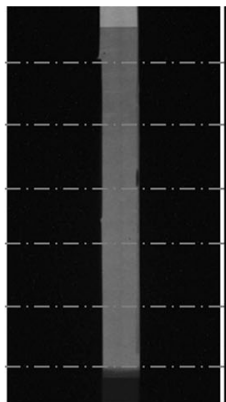

0 min

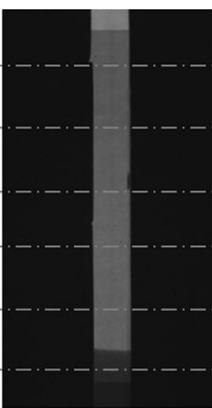

$5 \mathrm{~min}$

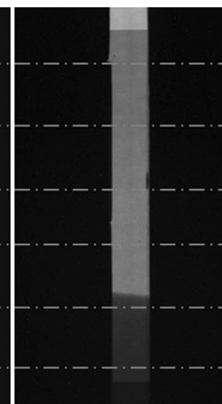

$33 \mathrm{~min}$

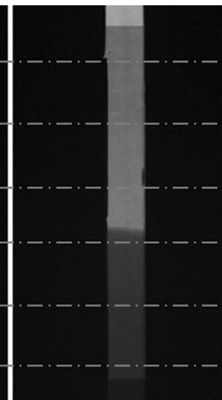

$84 \mathrm{~min}$

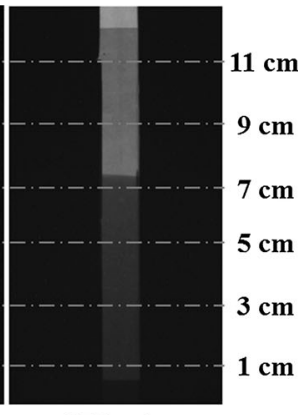

$156 \mathrm{~min}$

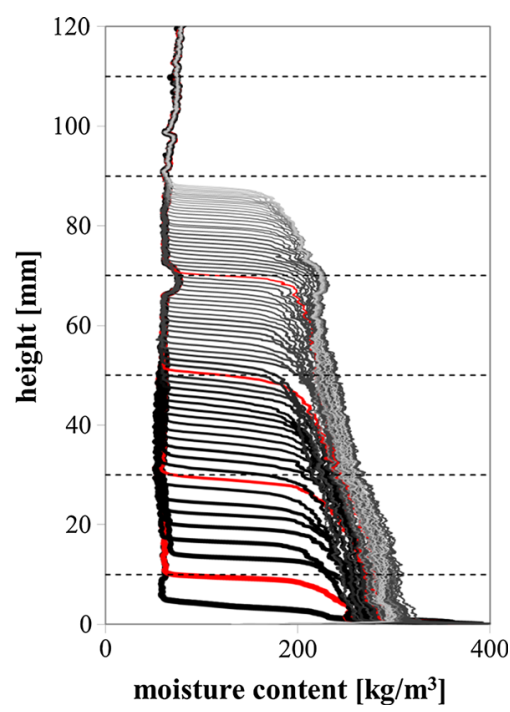

Fig. 8 Moisture content profiles in the brick sample during uptake as measured by neutron radiography over $264 \mathrm{~min}$. The profiles corresponding to time of water front arrival at each single contact position are highlighted in red. (Color figure online)

performed on the same samples, letting the current direction to alternate at each measurement. Applying the same liquid uptake procedure on a couple of samples, without neutron imaging this time, while alternating the direction of the current, resulted in steady readings at each position when it is reached by the liquid front. Repeating this procedure on the same sample, after drying, lead to somewhat lower readings (Fig. 9b). The reduction of quantity of salts dissolved in the wetting fluid explains in part the lower conductivity values measured during the last uptakes preformed.

As the readings seem to be affected by ionic displacement, which may occur at each liquid uptake event, an empirical correlation between the recorded electrical conductivity values and the moisture content
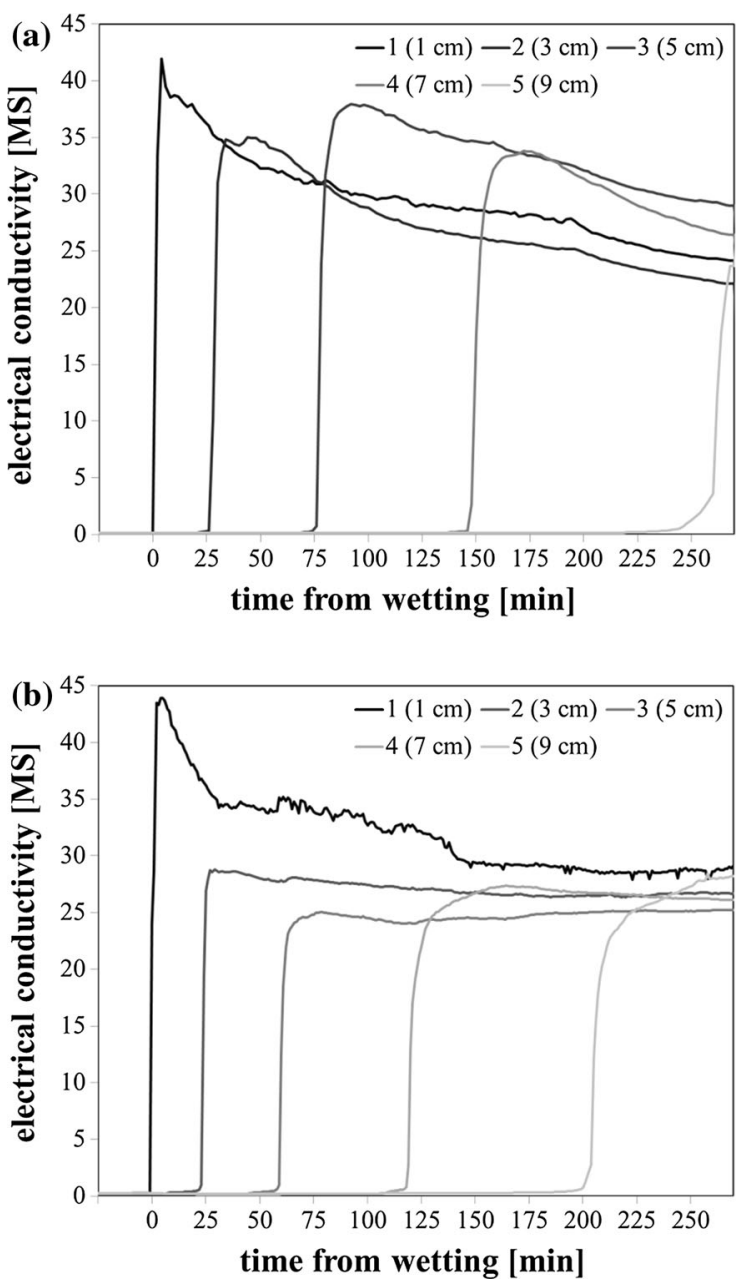

Fig. 9 a Electrical conductance versus time profiles measured in the brick sample during the uptake experiment documented with the neutron radiography. b Repeated experiment with alternating the direction of the electrical current

in the porous material could not be determined and a calibration of the sensor in terms of exact moisture content could not yet be achieved. 
Table 2 Comparison between image and sensor data, for the water front arrival times at each sensor position in the brick sensor

\begin{tabular}{llllll}
\hline & \multicolumn{5}{c}{ Water front time (min) } \\
\cline { 2 - 6 } Position & 1 & 2 & 3 & 4 & 5 \\
\hline Data from sensor analysis & 2 & 28 & 78 & 150 & 262 \\
Data from image analysis & 5 & 33 & 84 & 156 & \\
\hline
\end{tabular}

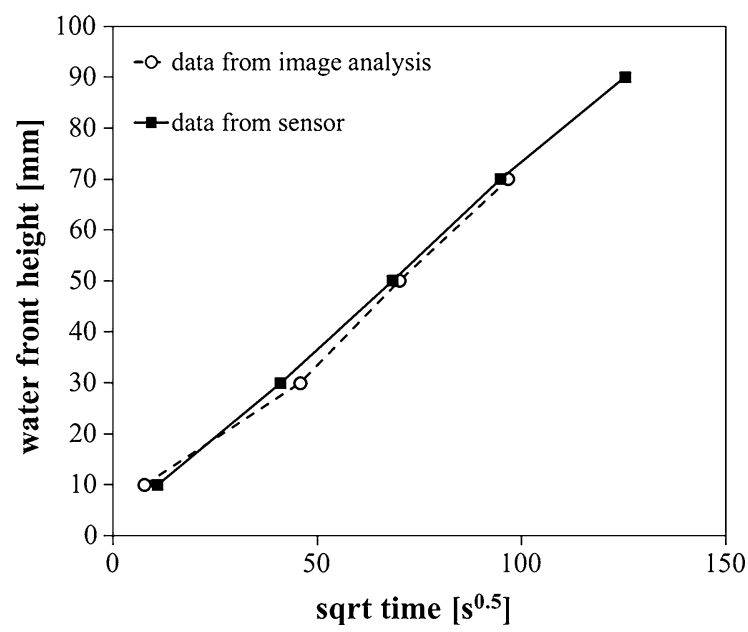

Fig. 10 Water front positions versus square root of arrival time measured by image analysis and contact pairs during the uptake experiment in the instrumented brick

\subsection{Sensors for wet applied materials}

We performed a similar comparative analysis between the moisture content profiles documented by neutron radiography and the electrical conductivity monitored by the sensors for the three samples of wet-applied building materials subjected to liquid uptake.

In the case of these materials, the delay of 30-48 s between water contact and the first neutron image prevents to document the initial steps of the much faster process. In fact, when the first radiographs are taken, the moisture front is already close to the maximum height it reaches during the uptake experiment, thus only the transport process through a limited number of sensor connections can be observed. Nevertheless the results provide information of the performance of the sensors.

The moisture content profiles generated by image analysis of the sample made of lime-cement mortar are plotted in Fig. 11a and refer to the first $80 \mathrm{~min}$ of the uptake experiment. The moisture content profiles show two clear steps in the lower part of the sample during the first part of the experiment. The capillary saturation zone is $4 \mathrm{~mm}$ high, while the wetted zone reaches up to $18 \mathrm{~mm}$ from the sample bottom. The contact between the sample and the water tank is lost quite soon due to the high amount of water uptaken by the material. Data obtained from the electrical conductivity sensor are analyzed and the times of electrical conductivity steps for the first six sensor positions are found in Table 3.

Profiles from the first $60 \mathrm{~min}$ of the uptake experiment for conventional cement mortar are presented in Fig. $11 \mathrm{~b}$. The first neutron image is taken after 3 min of wetting, by which time the water front has already reached the 5 th sensor position at a $26 \mathrm{~mm}$ height from the bottom. The following pictures are taken every $2 \mathrm{~min}$, but radiographs from 5 to $11 \mathrm{~min}$ have not been taken, therefore the profiles are estimated and plotted with dotted lines. Sensors undergo a step change at the first four contacts in the first minute from wetting. Also the sensor at position six shows electrical conductivity increasing after the first minute, while the sensor at position 5 reacts 2 min later. Positions from 7 to 11 react respectively from 2 to $6 \mathrm{~min}$ while the electrical conductivity measured in position 12 increases only after $18 \mathrm{~min}$, as summarized in Table 3.

The arrival times of the water front determined upon neutron images analysis and by the sensor for the lime-cement mortar and the conventional mortar are compared in Fig. 12. Results from the uptake experiment with the lime cement mortar fit well for the full uptake process and two uptake rates can be recognized both from sensor and image analysis data. For the sample with the conventional cement mortar, the uptake process is very fast at the beginning at the time resolution used for sensors readings and radiographs recording is too slow to give good description of the initial uptake process. The comparative analysis in Fig. 12b shows that the sensor reaction is slightly faster than the water front arrival uptake process detected by the neutron radiographs, especially in contact positions 7-10, while the arrival time at the highest monitored position is consistent between the two sets of data. This effect can be due to the presence of microcracks or not perfect contact between the mortar and the sensor that can cause local faster uptake rising close to the sensor. 
(a)

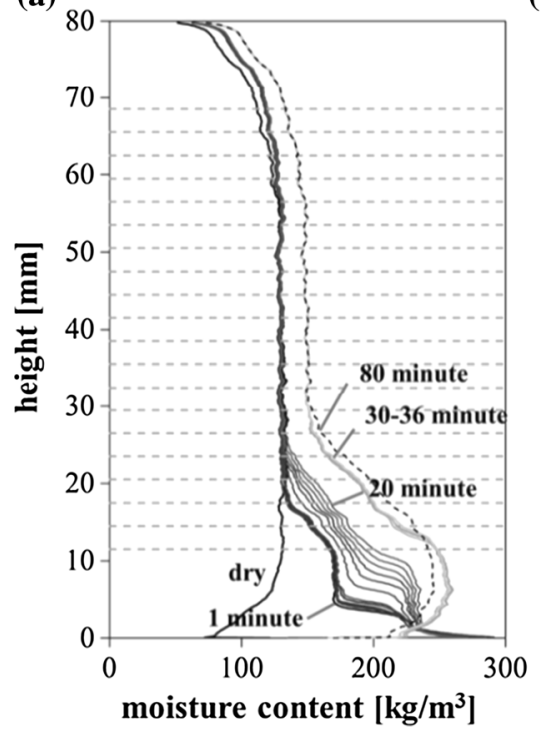

(b) 80

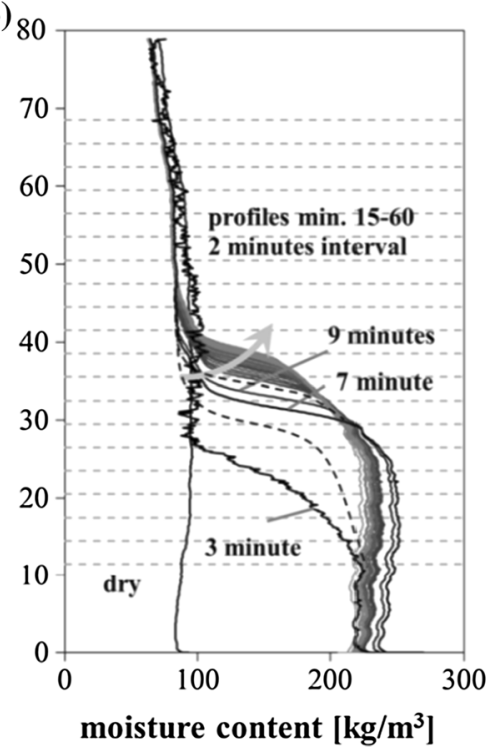

(c)

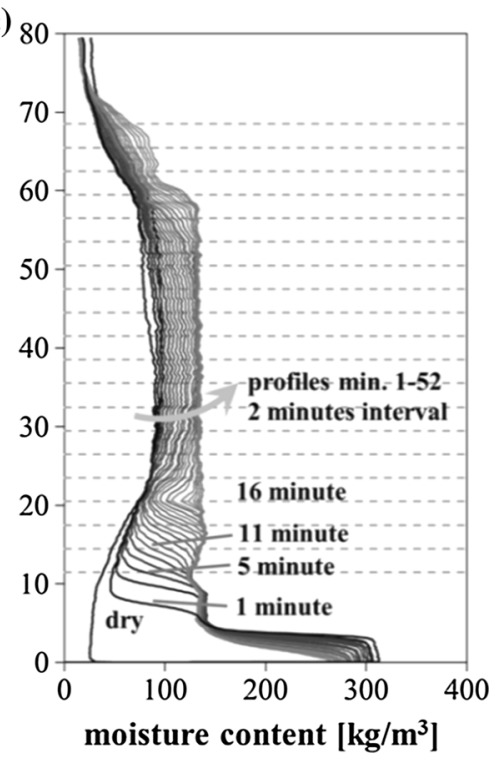

Fig. 11 Moisture content profiles as measured by neutron radiography of the samples made of lime-cement mortar (a), conventional cement mortar (b) and aerogel plaster (c) during the uptake experiment documented with the neutron radiography

Table 3 Comparison between image and sensor data, for the water front arrival times at each sensor position in the sensor

\begin{tabular}{llll}
\hline $\begin{array}{l}\text { Sensor } \\
\text { position }\end{array}$ & $\begin{array}{l}\text { Height } \\
(\mathrm{mm})\end{array}$ & $\begin{array}{l}\text { Lime-cement } \\
\text { mortar arrival } \\
\text { time (min) }\end{array}$ & $\begin{array}{l}\text { Cement mortar } \\
\text { arrival time } \\
(\mathrm{min})\end{array}$ \\
\hline 1 & 11 & 1 & 1 \\
2 & 14 & 1 & 3 \\
3 & 17 & 1 & 9 \\
4 & 20 & 1 & 15 \\
5 & 23 & 1 & 15 \\
6 & 26 & 2 & 27 \\
7 & 29 & 4 & 72 \\
8 & 32 & 5 & - \\
9 & 35 & 6 & - \\
10 & 38 & 7 & - \\
11 & 41 & 8 & - \\
12 & 44 & 18 & - \\
\hline
\end{tabular}

Results from the first hour of the uptake experiment for the sample with the aerogel plaster are presented in Fig. 11c. The initial state moisture content profile revealed that the sample was not homogenously conditioned in its central part. Nevertheless, in this experiment, the first picture is taken less than a minute after the wetting started and, given that the sorption process is slower compared to the two previous cement mortar samples, a better description of the water uptake is captured. In the first wet picture, the water front is approximately at $9 \mathrm{~mm}$ from the bottom surface and it reaches the first sensor position after $4 \mathrm{~min}$. From the profiles, a lower area of a thickness of 4-5 $\mathrm{mm}$ can be identified in its bottom part, where the water content decreases with time. This part corresponds to the lower part of the sample that is initially immersed in the water tank, which gradually empties during the uptake experiment. The electrical conductivity steps recorded by the sensor are not consistent with the image profiles. The first three contacts react immediately after the water contact is achieved. All other contacts show a reaction during the experiment but their time distribution is not consistent with their positions. In this case, a good agreement between sensor and neutron image data is not obtained. It proves that the sensor is not suitable for measurements with the aerogel plaster, probably due to the dimension of the aggregates that is similar to the holes of the sensor. The holes between the contacts must not be completely filled with the paste and the presence of void spaces prevent the good functioning of the sensor. The hydrophobicity of the aerogel grains further must increase these effects, influencing the water filling of pores close to the sensor contacts. 
Fig. 12 Water front positions versus square root of arrival time measured by image analysis and contact pairs during the uptake experiment with the limecement mortar (a) and the conventional cement mortar (b)

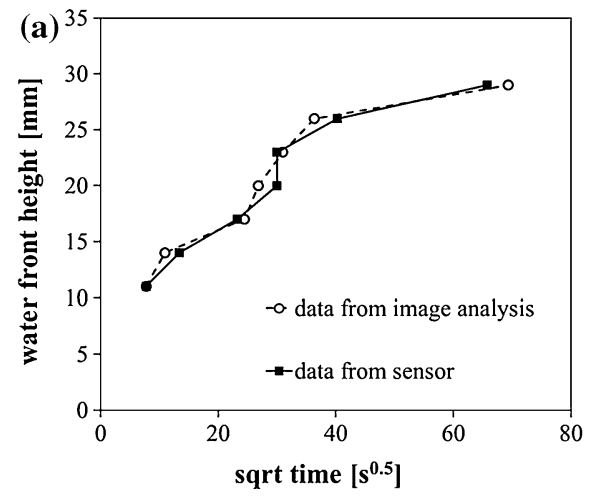

\section{Results discussion}

The proposed electrical conductivity sensors measure the electrical resistance of the material that lies between two tin contacts at different time steps. Upon arrival of the liquid front at the level of a contact pair, the electrical conductivity undergoes a step change. These step changes are regularly distributed over time in the different experiments and are consistent with the water front arrival times depicted by neutron radiography.

The temporal description by the sensors of the water front movement during water capillary uptake experiments is proved to be accurate. However a calibration allowing the measurement of absolute moisture content in each position has not yet been achieved. The absolute measured electrical conductivity can vary significantly the values recorded by the couple of contacts and also by the same contacts during different experiments. This can be due to some inhomogeneity in the material along the line connecting the two tin contacts that affects the measured conductivity values. For example, the presence of a big pore that is filled when the water front comes, can determine lower electrical conductivity in the dry state jumping to a very higher value when the pore is filled up with water.

From this initial but promising work, further optimization of the sensor is possible. In the bricks used in these experiments, the contacts are custom made. If they were industrially produced, a better performance could be expected. Bricks specially manufactured with lower content of salts could be selected to be used as sensors, in order to reduce the effect of movement of salts on the measurements. Also the shape of the sensors for mortar applications can be optimized to suite to the composition of the different mortars and renders and guarantee the formation of homogeneous material in the gaps between the electrical contacts. In particular the free space between the contacts can be enlarged and the construction of contacts can be improved.

The types of sensor proposed in this work are easily applicable in different positions of buildings, even deep in the structural components and they can be a useful, even though qualitative, instrument for long term monitoring in real applications. They allow continuous measurements over long periods without any destructive intervention. They can be employed in the monitoring of critical positions where penetration of liquid water is expected, such as rising damp effects in basement structures and critical positions such as flat roofs, or wall parts close to windows and doors. Additionally they can be used for monitoring the drying of materials during the construction, especially when the full drying of wet-applied layers is necessary before the application of finishing layers improving the quality of the building process. Sensors can also be applied in renovation intervention to monitor hygrothermal changes due to the introduction of new materials, or in critical position to check possible leakages or water rising phenomena. A first example of such use is described in [8]. By connecting the sensors to a data logger outside the wall, data can be recorded at different times after the construction process without any destructive action on the building. These sensors are low cost, easy to produce and their shape can easily be adapted to monitor different positions. Even if the measurement of the absolute value of the moisture content in the most internal construction layer cannot be as precise as with other destructive investigation technique, i.e. gravimetry 
measurements, the system allows repeatable and continuous monitoring of relative changes due to the transport of liquid water through the building component.

\section{Conclusions}

In this paper we have described the design, the construction and the validation experimental campaign of moisture content sensors for building materials based on the measurement of electrical conductivity. Special samples of brick and wet applied mortars have been built and a capillary uptake experiment has been documented with neutron radiographs. A strong correlation is found between results obtained with the different approaches, especially dealing with water front travelling time. We can conclude that the measurement system proposed can be reliable for the investigation of water penetration in building materials.

The sensors allow continuous monitoring with high space and time resolution that can be adapted as required by specific applications. Experiments show that the liquid uptake phenomenon can be adequately captured and results from almost all material samples show clear steps in the measured electrical conductivity as the water front travels through the contacts positions. The behavior of the sensors in monitoring drying phases needs additional and more extensive investigations. Such system can be applied for the long-term performance assessment of buildings, assessment of retrofit and renovation strategies of building envelopes, the evaluation of the need of dehumidification treatments, etc.

\section{References}

1. Archie GE (1942) The electrical resistivity log a s an aid in determining some reservoir characteristics. Pet Trans AIME 146:54-62

2. Berliner MA (1980) Feuchtemessung. VEB Verlag Technik, Berlin

3. Birlea NMC, Culea EN (2012) Electrical methods for testing buildings and construction materials. In: Proceedings of first international conference for $\mathrm{PhD}$ students in Civil Engineering, CE-PhD, 4-7 Nov Cluj_Napoca, Romania

4. Courtois, A., Taillade, F., Moreau, G., Clauzon, T., Skoczylas, F., Masson, B, 2013, Water content monitoring for nuclear concrete buildings: needs, feedback and perspectives, PoromechanicsV, ASCE

5. Daniels DJ (2004) Ground-penetrating radar. Institute of Electrical Engineers, London

6. Dean TJ, Bell JP, Baty AJB (1987) Soil moisture measurement by an improved capacitance technique. Sensor design and performance. J Hydrol 93:67-78

7. Ewing RP, Hunt AG (2006) Dependence of the electrical conductivity on saturation in real porous media. Vadose Zone J 5:731-741

8. Guizzardi M (2014) Hygrothermal performance assessment of novel interior insulation solutions. Ph.D. Thesis, Chair of Building Physics, ETH Zürich

9. Gummerson RJ, Hall C, Hoff WD (1980) Water movement in porous building materials-II. Hydraulic Suction and Sorptivity of Brick and Other Masonry Materials. Build Environ 15:101-108

10. Hassanein R (2006) Correction methods for the quantitative evaluation of thermal neutron tomography. Ph.D. Thesis, ETH Zürich, Switzerland

11. Hassanein R, Meyer H, Carminati A, Estermann M, Lehmann E, Vontobel P (2006) Investigation of water imbibition in porous stone by thermal neutron radiography. J Phys D: Appl Phys 39(19):4284-4291

12. Krus M (1995) Moisture transport and storage coefficients of porous mineral building materials. Ph.D. Thesis, Universität Stuttgart, Germany

13. Kupfer K (1990) Feuchtemessung an Zuschlagstoffen für die Betonherstennung unter Verwendung der Mikrowellenmesstechnik. Dissertion, Hochschule für Architektur und Bauwesen Wismar

14. Leschnik W (1999) Feuchtemessung an BaustoffenZwischen Klassik und Moderne, Feuchtetag '99. Umwelt Meßverfahren Anwendungen, 7/8 Oktober 1999, BAM, Berlin

15. McCarter WJ, Vennesland O (2004) Sensor systems for use in reinforced concrete structures. Contr Build Mater 18:351-358

16. Pel L (1995) Moisture transport in porous materials. $\mathrm{PhD}$ Thesis, TU/e, Eindhoven, The Netherlands

17. Plack GV (1987) Resistivity characteristcs of geological targets. Electromag Methods Appl Geophys 1 (Theory), p 1351

18. Rajabipour F. 2006. Insitu electrical sensing and material health monitoring in concrete structures. PhD thesis, Publication Number: AAI3259972, Purdue University

19. Rajabipour F, Weiss J (2007) Electrical conductivity of drying cement paste. Mater Struct 40:1143-1160

20. Raupach M, Schiess1 P (2001) Macrocell sensor systems for monitoring of the corrosion risk of the reinforcement in concrete structure. NDT E Int 34:435-442

21. Sandrolini F, Franzoni E (2006) An operative protocol for reliable measurements of moisture in porous materials of ancient buildings. Build Environ 41:1372-1980

22. Sedighi Gilani M, Griffa M, Mannes D, Lehmann E, Carmeliet J, Derome D (2012) Visualization and quantification of liquid water transport in softwood by means of neutron radiography. Int J Heat Mass Transf 55:6211-6221 